\title{
Site-Specific and Temporal Effects of Apraglutide, a Novel Long-Acting Glucagon-Like Peptide-2 Receptor Agonist, on Intestinal Growth in Mice
}

\author{
S.E. Martchenko, M.E. Sweeney, V. Dimitriadou, J.A. Murray, and (DP.L. Brubaker
}

Departments of Physiology (S.E.M., M.E.S., P.L.B.) and Medicine (P.L.B.), University of Toronto, Toronto, Ontario, Canada; VectivBio AG, Basel, Switzerland (V.D.); and Division of Gastroenterology Mayo Clinic, Rochester, Minnesota (J.A.M.)

Received November 13, 2019; accepted March 5, 2020

\begin{abstract}
Long-acting glucagon-like peptide-2 receptor (GLP-2R) agonists are well-established to increase intestinal growth in rodents and, most notably, humans with short bowel syndrome. Most of the trophic effects of GLP-2R agonists are reported to be mediated through increased growth of the crypt-villus axis, resulting in enhanced mucosal mass and improved intestinal function. The present study examined the effects of apraglutide, a novel GLP$2 \mathrm{R}$ agonist, on the growth of the small intestine and colon after 3 , 7 , and 10 weeks of treatment in male and female mice. Apraglutide ( $3 \mathrm{mg} / \mathrm{kg}$; three times per week) significantly increased small intestinal weight $(P<0.001)$ and length $(P<0.001)$ after 3 weeks of administration, with a further increase in effectiveness after 10 weeks $(P<0.01)$. Crypt depth and villus height were both markedly increased after 3 weeks of apraglutide administration $(P<0.001)$ but did not show any further increase with duration of
\end{abstract}

treatment, whereas crypt number and intestinal circumference were increased after 7 and 10 weeks $(P<0.01)$ but not after 3 weeks of apraglutide treatment. Both the weight and the length of the colon were also enhanced by apraglutide treatment for 3 weeks $(P<0.001)$, and these effects were maintained but did not improve further with continued apraglutide administration. The results of this study demonstrate that the novel, long-acting GLP-2R agonist, apraglutide, demonstrates an unexpected marked ability to increase intestinal length as well as exert timeand location-dependent specificity in its intestinotrophic actions.

\section{SIGNIFICANCE STATEMENT}

The novel long-acting glucagon-like peptide 2 receptor agonist, apraglutide, enhances intestinal weight as well as intestinal length in a time- and site-dependent fashion.

\section{Introduction}

Long-acting, dipeptidylpeptidase IV-resistant glucagonlike peptide (GLP)-2 receptor (GLP-2R) agonists, most notably human (h) [Gly2]GLP-2(3-33) are well-established to increase intestinal growth in animals as well as in humans (Brubaker, 2018). These actions are mediated through increased proliferation and decreased apoptosis of the intestinal epithelial cells, resulting in expansion of both crypt depth and villus height (Drucker et al., 1996; Tsai et al., 1997; Dubé et al., 2006; Rowland et al., 2011). Although GLP-2R expression is greatest in the midsmall intestine (Munroe et al., 1999), trophic effects of h[Gly2]GLP-2(3-33) are observed throughout the small and

This work was supported by operating grants from GLyPharma Therapeutics, Inc. (now VectivBio AG) and from the Canadian Institutes of Health Research [Grant PJT-14853]. M.E.S. was supported by summer studentships from the Canadian Association of Gastroenterology and the University of Toronto Research Opportunity Program, and P.L.B. was supported by the Canada Research Chairs Program.

SEW, MES and PLB have no conflicts of interest to declare. VD is a previous employee of GLyPharma Therapeutic, Inc. JAM has grants from the NIH, Crohn's and Colitis Foundation of America, Immunogenix, Allakos, DBV Technologies, and Cour. He has consulted for Vibrant Technologies, and Actobiotics., Innovate, Kanyos, Calypso, and Teva Pharma, and holds a patent US8617536, prevotella histicola, licensed to Evelo.

https://doi.org/10.1124/jpet.119.263947. large intestine (Drucker et al., 1997; Dubé et al., 2006; Yusta et al., 2012). However, the growth effects of h[Gly2]GLP-2(333) in normal rodents appear to be largely restricted to the intestinal mucosa, with variable effects on small intestinal length (i.e., 0\%-5\%) and no change in colonic length reported to date (Drucker et al., 1997; Hadjiyanni et al., 2009; Yusta et al., 2012; Austin et al., 2015).

Strikingly, extremely few studies have examined changes in intestinal growth responses to GLP-2 over time. In one early study on CD-1 mice, the increases in small intestinal weight and crypt-villus height induced by native GLP-2 appeared to be greater after 12, as compared with 4 and 8 , weeks of treatment (Tsai et al., 1997). Although the magnitude of the responses was not directly compared between the different time points, absolute small intestinal weight was increased by $\sim 20 \%$ as compared with $\sim 60 \%$ after 4 and 12 weeks of treatment, respectively. Similarly, crypt-villus height in the proximal jejunum appeared to increase by $\sim 60 \%$ after 4 weeks and by $\sim 75 \%$ after 12 weeks. A second study utilizing h[Gly2] GLP-2(3-33) in the same mouse strain demonstrated a 16\%-20\% greater increase in small intestinal and colonic weights after 30 days of treatment as compared with 10 days but found that the stimulated increase in crypt-villus height $(37 \%-75 \%)$ did not change beyond the 10-day time point; 
again, none of these apparent differences were compared statistically (Kissow et al., 2012). We further noted that neither study reported any findings on intestinal length.

In the present preclinical study, we have examined the trophic effects of a novel long-acting human GLP-2R agonist, apraglutide (Wiśniewski et al., 2016), on small intestinal and colonic weight and length as well as small intestinal cryptvillus height and crypt number in mice, directly comparing the temporal responses to chronic treatment of 3, 7, and 10 weeks. Apraglutide is a new GLP-2 analog that is distinguished from other GLP-2 analogs, such as teduglutide (Revestive/Gattex, Shire Inc.), because of its very low clearance, long elimination half-life (30 hours), and high plasma protein binding [(Hargrove et al., 2020) and unpublished data]. In humans, such long half-life after subcutaneous administration () (Bolognani et al, 2019) allows only once-weekly treatment, and apraglutide is being developed for use in short bowel syndrome (SBS) as a once-a-week treatment (phase 2 trials completed in SBS patients: NCT03408132, NCT03415594). In addition, apraglutide has previously been administered in healthy volunteers (phase 1 trial successfully completed in Canada). Data from these studies show that apraglutide is generally welltolerated in healthy volunteers and has an overall positive safety profile (Bolognani et al., 2019). The findings of the present study, unexpectedly, demonstrate site-specific as well as time-dependent actions of apraglutide on both intestinal epithelial and linear growth.

\section{Materials and Methods}

NOD/DQ8 mice were obtained from Dr. J.A. Murray (Mayo Clinic College of Medicine, Rochester, MN) courtesy of Dr. E.F. Verdu (McMaster University, Hamilton, ON) and were then bred in-house. Historically, this murine strain was used to establish a model of the gluten hypersensitivity seen in celiac disease (Marietta et al., 2004; Galipeau et al., 2011) but, once at the University of Toronto, these mice did not demonstrate any signs of gluten-induced enteropathy (unpublished data). Hence, these mice were thereafter used as a physiologic model to study the long-term actions of apraglutide, with sensitization and gluten (Marietta et al., 2004; Galipeau et al., 2011) administered to both male and female littermates (6-8 weeks of age; $n=4-8$ each, to make $n=8$ for the 3 -week study and $n=13$ for remaining studies) and treatment with either vehicle $(4.5 \%$ D-mannitol, $20 \mathrm{mM}$ glycine, $20 \mathrm{mM}$ L-histidine, $\mathrm{pH}$ 8.6-8.8; control) or apraglutide $(3 \mathrm{mg} / \mathrm{kg}$ or $\sim 1000 \mathrm{nmol} / \mathrm{kg})$. The selection of the dose of apraglutide was based on previous studies showing equivalent activity on small intestinal weight for doses up to $25 \mathrm{mg} / \mathrm{kg}$ in rats and up to $20 \mathrm{mg} / \mathrm{kg}$ in mice. Vehicle and apraglutide were administered by subcutaneous injection three times per week for 3,7 , or 10 weeks, and this was followed by a single injection 24 hours prior to euthanization. The frequency of administration was based on the previous pharmacokinetic studies conducted in adult rats (225-250 g) (Hargrove et al., 2020 ) and in adult mice (7-10 weeks old or $21-26 \mathrm{~g})$. In mice, specifically, the elimination $t_{1 / 2}$ was in the range of $6-11$ hours with an accumulation ratio of 1.6 observed in male animals but not in female animals (unpublished data). Additional studies have demonstrated no effect of age on the intestinotrophic effects of GLP-2 in mice (Tsai et al., 1997). Apraglutide is a 33-amino-acid peptide with a molecular structure based on the sequence of GLP-2 but designed to optimize pharmacological activity while increasing the half-life versus other long-acting GLP-2 analogs. The compound provided by the sponsor, GLyPharma Therapeutic Inc. (Montreal, Canada), had a purity of $>90 \%$; its composition was fully characterized, and no known active impurities were detected. All animal protocols were approved by the Animal Care Committee of the University of Toronto, and all procedures were conducted in accordance with the regulations of the Canadian Council on Animal Care.

Mice were weighed on day 1 and then weekly throughout the study. After euthanasia with isoflurane, the small intestine (excluding $10 \mathrm{~cm}$ of jejunum, which was removed for other analyses) and colon were collected, rinsed with PBS, and weighed, and their lengths were measured under constant tension using a 2.8-g weight. Twocentimeter sections of the duodenum were collected into $4 \%$ formalin for paraffin embedding, sectioning, and staining with hematoxylin and eosin (Pathology Research Program, University Health Network, Toronto, ON). For each mouse, a minimum of 20 well-oriented villi and 20 crypts were measured on a Zeiss Axiovision microscope with AxioPlan software to make $n=1$ (Dubé et al., 2006; Rowland et al., 2011; Dong et al., 2014; Austin et al., 2015). A subgroup of the sections (from both males and females) was also examined for crypt number per intestinal cross-section, counting well-oriented crypts only, as well as for cross-sectional circumference $(n=3-6)$.

Data are shown as mean \pm S.E.M. for the absolute values as well as for the changes in apraglutide-treated animals relative to the timeappropriate vehicle control group. Data were compared by one- or twoway ANOVA followed by Tukey's Honest Significant Difference post hoc analysis, as appropriate. No significant differences were detected for either intestinal weights or lengths by sex, although males had significantly greater body weights, as expected, at 3 and 7 weeks $(P<$ 0.05 ). As a result, data from both sexes were combined for all further analyses.

\section{Results}

Absolute body weights of vehicle-treated mice and the weights of apraglutide-treated animals relative to vehicle controls did not change over time and were not different between treatment groups (Fig. 1A). Absolute small intestinal weights also did not change significantly over time in vehicletreated mice. However, apraglutide significantly increased small intestinal weight by $80 \%$ after 3 weeks of treatment $(P<0.001$; Fig. 1B). Interestingly, the magnitude of this effect increased in a time-dependent fashion, with a further increase of $51 \%$ after 10 weeks of treatment reaching $231 \%$ of vehicle control $(P<0.05-0.01$ vs. 7 and 3 weeks, respectively). Similarly, small intestinal length did not differ by time in vehicle animals but was increased by $13 \%$ after 3 weeks of apraglutide treatment $(P<0.05)$, an effect that increased by an additional $15 \%$ after 10 weeks of treatment $(P<0.05$ vs. 3 weeks; Fig. 1C). The trophic $(P<0.001)$ and time-dependent $(P<0.05-0.01)$ actions of apraglutide were also observed when small intestinal weight was normalized to body weight after 3 weeks of treatment (increased by $33 \%, P<0.001$ ) and increased by a further $20 \%$ and $35 \%$ at 10 weeks versus 3 and 7 weeks of treatment, respectively $(P<0.05-0.01$; Fig. 1D). These changes indicate the specificity of the growth effects of apraglutide for the small bowel. Furthermore, the timedependent increases in apraglutide-induced small intestinal weight were maintained when the data were normalized to small intestinal length $(P<0.001$ for treatment effect; $P<$ 0.05 for time effect; Fig. 1E), demonstrating that the trophic effects were induced per unit length of small intestine (increased by $59 \%$ after 3 weeks, $P<0.001$, and by a further $13 \%$ after 10 weeks of treatment, $P<0.05$ ) in addition to the increase in overall length.

To examine potential mechanisms underlying the increase in small intestinal mass with apraglutide over time, duodenal structure was analyzed morphometrically. No differences in villus height, crypt depth, crypt number per cross-section, or 
A Body weight $\square \mathrm{VEH}$
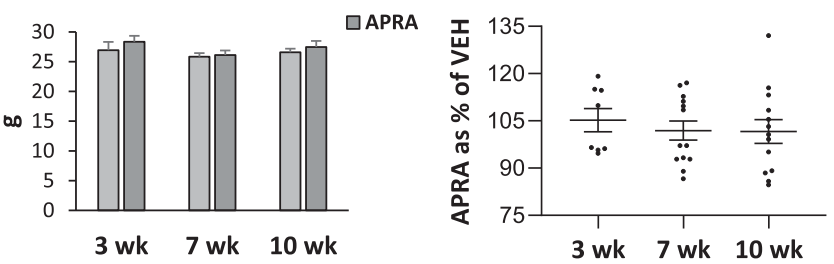

B


C SI length $\quad \square$ VEH



D SI weight/body weight
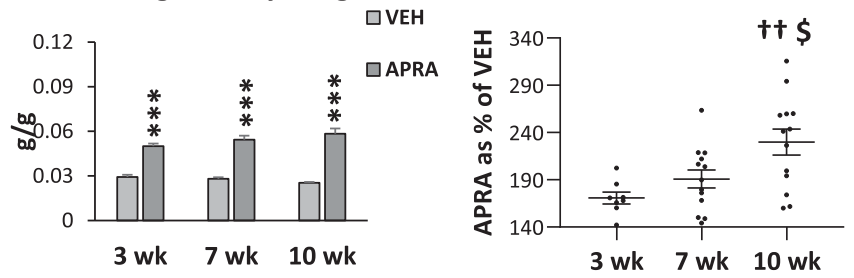

E SI weight/length $\square$ VEH
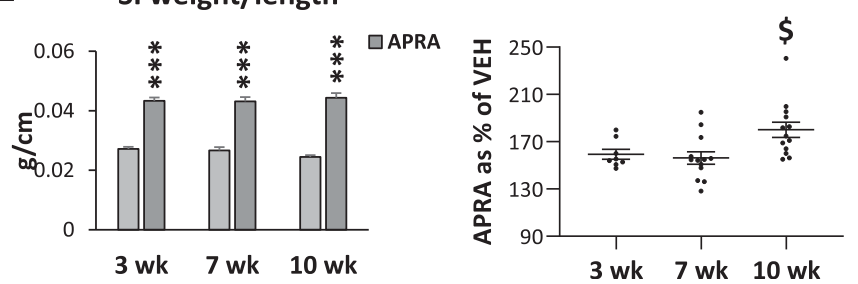

Fig. 1. Body weight (A) and small intestinal (SI) weight (B), length (C), weight/body weight (D) and weight/length (E) in vehicle (VEH)- and apraglutide (APRA)-treated mice; all data are shown as mean \pm S.E.M. (Left) Absolute changes $(* P<0.05 ; * * * P<0.001)$; (Right) percent responses ( ${ }^{\dagger} P<0.05$; ${ }^{\dagger} P<0.01$ vs. 3 weeks; ${ }^{\$} P<0.05$ vs. 7 weeks).

circumference were observed over time in vehicle-treated mice (Fig. 2). Apraglutide increased both villus height and crypt depth after 3 weeks of treatment (by $36 \%$ and $37 \%$, respectively; $P<0.001$ ). However, unexpectedly, the overall magnitude of the stimulation was not further increased after 7 or 10 weeks of treatment compared with the 3 -week time point. In contrast, neither crypt number nor intestinal circumference was significantly increased after 3 weeks of apraglutide administration, but both were markedly enhanced after 7 weeks, as well as 10 weeks, of treatment (by 16\%-18\% and $22 \%-23 \%$, respectively, $P<0.01$ ).

Finally, colon weight unexpectedly decreased over time in vehicle-treated animals (by 16\%-19\%; $P<0.05$ for 3 weeks vs.
7 and 10 weeks), possibly because of the strain of mice used. Notwithstanding, and in marked contrast to the findings in the small intestine, apraglutide increased absolute colon weight and length as well as normalized colon weight (per body weight and per colon length) after 3 weeks of treatment (by $40 \%, 21 \%$, 33\%, and 16\%, respectively, $P<0.05-0.001$; Fig. 3), but no further increases in any of the colonic responses to apraglutide were observed after either 7 or 10 weeks of administration.

\section{Discussion}

Both synthetic h[Gly2]GLP-2(3-33) and the recombinant GLP-2R agonist, teduglutide, have been well-established to increase the growth and function of the intestines in multiple species, including, importantly, humans with short bowel syndrome (Drucker et al., 1996, 1997; Tsai et al., 1997; Dubé et al., 2006; Hadjiyanni et al., 2009; Rowland et al., 2011; Yusta et al., 2012; Jeppesen, 2013; Austin et al., 2015; Brubaker, 2018). The results of the present study demonstrate biologic effectiveness of a novel, long-acting GLP-2 analog, apraglutide, to enhance the growth of both the small intestine and the colon in a preclinical animal model.

Previous studies have shown sex- and age-independent maintenance of the trophic effects of native GLP-2 on small intestinal weight and crypt-villus height over 4-12 weeks of treatment in mice (Tsai et al., 1997). However, few studies have examined the actions of either GLP-2 or of GLP-2R agonists over time in both the small intestine and colon, and none of these have conducted direct analyses of any timedependent responses (Tsai et al., 1997; Kissow et al., 2012). Strikingly, the intestinotrophic effects of apraglutide were found to be temporally mediated in a site-specific fashion, such that progressive increases were observed over 3-10 weeks in both the weight and the length of the small intestine but not of the colon, which achieved maximal growth after only 3 weeks of treatment.

The specificity of the actions of apraglutide for intestinal growth was demonstrated by the lack of change in body weight in treated animals (other than a small, nonsignificant increase commensurate with the increased weight of the bowel), as previously reported (Brubaker et al., 1997). Furthermore, although GLP-2 facilitates digestive and absorptive functions in normal animals, these actions are not paralleled by an increase in food intake (Brubaker et al., 1997); hence, the efficiency of nutrient handling is improved by GLP-2 treatment without associated changes in energy homeostasis. In contrast, GLP-2R agonist therapy is associated with weight gain in humans with short bowel syndrome, likely because of the improved capacity for enteral nutrition (Jeppesen et al., 2001).

How the temporal changes in small intestinal weight are exerted by apraglutide appear to be complex. Consistent with the results of a previous study on h[Gly2]GLP-2(3-33), although not on native GLP-2, in CD-1 mice (Tsai et al., 1997; Kissow et al., 2012), the increased weight over time was not associated with additional growth of the villus-crypt axis beyond that observed at the 3-week time point. However, despite a previous report that GLP-2R agonist therapy does not appear to affect crypt number (Koehler et al., 2015), the results of the present study clearly demonstrate not only increased crypt number but also increased cross-sectional 

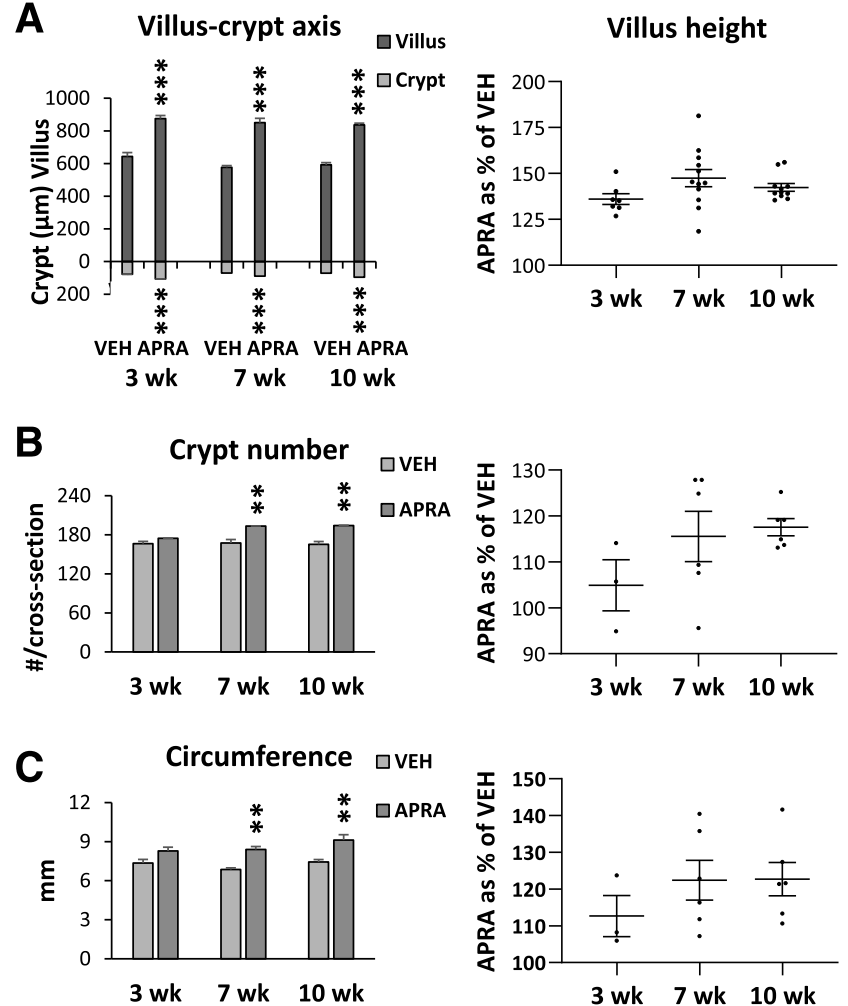

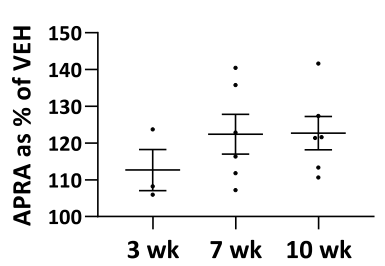

Villus height

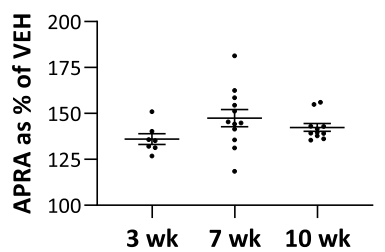

3 wk $7 w k 10 w k$

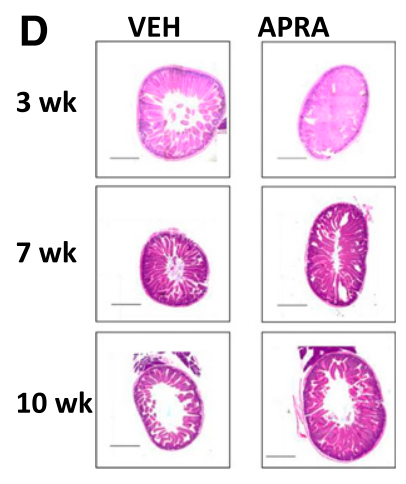

Fig. 2. Duodenal villus height and crypt depth (A), crypt number per cross-section (B), cross-sectional circumference (C), and representative photomicrographs (scale bar, $1 \mathrm{~mm}$ ); (D) in vehicle (VEH)- and apraglutide (APRA)-treated mice; all data are shown as mean \pm S.E.M. (Left) Absolute changes (** $\mathrm{P}<0.01 ; * * * P<$ 0.001); (Right) percent responses $\left({ }^{\dagger} P<\right.$ 0.05 vs. 3 weeks). circumference after 7 and 10 weeks of apraglutide treatment. These findings suggest that there may be limits to the vertical growth of the crypt-villus axis that, in the setting of continued trophic stimulation, may induce crypt fission. Although this process has been described in the normal adult mouse intestine, the regulation of crypt fission remains poorly understood (Bruens et al., 2017). However, importantly, even in the setting of profound stimulation of intestinal growth by GLP-2, no changes in the normal epithelial cell differentiation pathway have been noted and, indeed, epithelial cell width has been reported to decrease (Benjamin et al., 2000; Lee et al., 2012). Finally, at least part of the apraglutide-induced intestinal growth was achieved by increased length of the small bowel, because small intestinal weight gain independent of body weight increased by $29 \%$ after 10 weeks, but weight gain independent of length increased by only $13 \%$ during the same period; these findings suggest that $16 \%$ of the increase in small intestinal weight with apraglutide treatment over time occurred consequent to its increased length. Indeed, the $13 \%$ increase in small intestinal length observed after only 3 weeks of administration of apraglutide is further remarkable given the limited effect of h[Gly2]GLP-2(3-33) reported for this parameter in rodents (Drucker et al., 1997; Hadjiyanni et al., 2009; Yusta et al., 2012; Austin et al., 2015). Apraglutide has also been demonstrated to increase small intestinal length in a neonatal piglet model of short bowel syndrome, leading to the suggestion that the trophic actions of apraglutide may enhance adaptation and weaning from parenteral nutrition in young children with short bowel syndrome (Slim et al., 2019). Whether enhanced intestinal length will be observed in humans treated with GLP-2R agonists remains to be determined. Nonetheless, increased citrulline levels, which are commonly used as a measure of total mucosal mass that also reflects intestinal length, have been reported in patients with short bowel syndrome and Crohn's disease in response to h[Gly2]GLP-2(3-33) (teduglutide) treatment (Crenn et al., 2000; Buchman et al., 2010; Jeppesen et al., 2011, 2012; Seidner et al., 2015), although the sensitivity of this marker has recently been questioned (Lansing et al., 2019).

Although apraglutide exerted the anticipated effects to enhance colonic weight (Drucker et al., 1997; Litvak et al., 1998; Dubé et al., 2006), unexpected large increases in colonic length were also observed. As for the small intestine, these trophic actions of apraglutide were independent of changes in body weight. These findings are consistent with high levels of expression of the GLP-2R in the rat and human colon (Munroe et al., 1999; Yusta et al., 2000), although, to date, no studies have examined the mechanism of action of GLP-2 to increase the length of either the small or large intestine. Importantly, the other effects of apraglutide on the colon were not only generally smaller than those on the small intestine, but all of the changes were also temporally limited, such that maximal growth was achieved after only 3 weeks of treatment. Of equal importance, given that chronic administration of long-acting GLP-2 analogs has been linked to the progression of chemically induced intestinal tumors in rodents (Iakoubov et al., 2009; Trivedi et al., 2012), visual examination of the large intestines in the apraglutide-treated cohort did not reveal any gross evidence of abnormal growth. Notwithstanding, although there is currently a lack of evidence for such effects of GLP-2R agonists in humans (Orhan et al., 2018; Ring et al., 2018), and ongoing monitoring of patients remains prudent.

\section{Clinical Perspective}

Few agents have been demonstrated to be effective in increasing both intestinal mass and length without whole-body 
A Colon weight $\quad$ QVEH
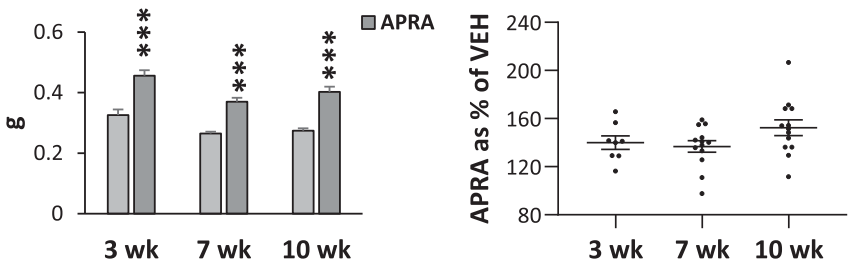

B


Fig. 3. Colon weight (A), length (B), weight per body weight (C), and weight per length (D) in vehicle (VEH)- and apraglutide (APRA)-treated mice; all data are shown as mean \pm S.E.M. (Left) Absolute changes $(* P<$ 0.05 ; ** $\mathrm{P}<0.01$; *** $P<0.001$ ); (Right) percent responses.

side effects. For example, although transgenic overexpression of growth hormone or insulin-like growth factor-1 causes increases in both parameters, these hormones also induce parallel increases in body weight of $91 \%$ and $37 \%$, respectively (Ulshen et al., 1993; Ohneda et al., 1997). Furthermore, h [Gly2]GLP-2(3-33) has only limited actions to increase mucosal length despite profound stimulatory effects on mucosal growth (Drucker et al., 1997; Hadjiyanni et al., 2009; Yusta et al., 2012; Austin et al., 2015). Notwithstanding, residual intestinal length has been positively correlated to maintenance of the beneficial effects of teduglutide for 12 months after withdrawal in adults with SBS (Compher et al., 2011), suggesting that this is a desirable outcome of treatment. Importantly, the finding of increased small intestinal and colonic lengths in the present study in mice without bowel resection is consistent with a previous report on residual bowel lengthening with apraglutide in neonatal piglet model of SBS. Although increases in intestinal length have not been reported in response to teduglutide treatment of adults, it has been suggested that any increase in bowel length may be of benefit to the pediatric SBS population (Slim et al., 2019). Furthermore, it remains unclear as to whether teduglutide treatment affects the growth of the colon in humans. Future studies in humans to determine these specific parameters of intestinal growth are clearly required to fully determine the translational relevance of the current findings in mice. However, given that many previous preclinical studies on the intestinotrophic effects of GLP-2 in the normal murine intestine have been successfully translated to humans, these findings suggest that this novel action of apraglutide may benefit patients through expansion of the residual, healthy tissue. Finally, the half-life of apraglutide is prolonged $\left(t_{1 / 2}=\right.$ 30 hours) as compared with both native GLP-2 and h[Gly2] GLP-2(3-33) ( 6 minutes and 1-3 hours, respectively), which is due to both dipeptidylpeptidase IV-resistance and binding to proteins in the circulation (99.8\%) (Tavares et al., 2000; Marier et al., 2010; Slim et al., 2019). As a consequence, apraglutide was administered only three times per week in the present study, as compared with the current requirement for daily treatment with h[Gly2]GLP-2(3-33) and teduglutide, although it is acknowledged that the dose of apraglutide used in the current study on mice is $\sim 3$-times greater than that currently being tested for human use () (Bolognani et al, 2019). The marked effectiveness of apraglutide combined with a reduced burden for administration may, ultimately, enhance patient compliance in the clinical setting.

In summary, the results of the present preclinical study demonstrate highly specific and progressive actions of apraglutide to increase intestinal weight and length in normal mice. Unexpectedly, the trophic effects of apraglutide on the small bowel were found to increase over time, whereas those on the colon were temporally restricted. These preclinical findings provide support for further analyses of the effectiveness of apraglutide to enhance structure and function in models of human intestinal disease.

\section{Acknowledgments}

The authors are grateful to Dr. E.F. Verdu (McMaster University, Ontario, Canada) for the generous gift of the mice and to Dr. Federico Bolognani (VectivBio AG) for helpful comments on the manuscript.

\section{Authorship Contributions}

Participated in research design: Martchenko, Dimitriadou, Brubaker.

Conducted experiments: Marchenko, Sweeney.

Contributed new reagents or analytic tools: Dimitriadou, Murray. Performed data analysis: Martchenko, Sweeney, Brubaker.

Wrote or contributed to the writing of the manuscript: Martchenko, Sweeney, Dimitriadou, Murray, Brubaker.

\section{References}

Austin K, Imam NA, Pintar JE, and Brubaker PL (2015) IGF binding protein-4 is required for the growth effects of glucagon-like peptide-2 in murine intestine. Endocrinology 156:429-436.

Benjamin MA, McKay DM, Yang PC, Cameron H, and Perdue MH (2000) Glucagonlike peptide-2 enhances intestinal epithelial barrier function of both transcellular and paracellular pathways in the mouse. Gut 47:112-119.

Bolognani F, Dimitriadou V, Jean Bourgouin MP, Stavenhagen J, Porter R, Meyer C, and Santarelli L (2019) Pharmacokinetics and pharmacodynamics of glucagon-like peptide (GLP-2) analog apraglutide (FE 203799) in adult healthy volunteers: results of a Phase I trial. Transplantation 103: S128.0041-1337

Brubaker PL (2018) Glucagon-like peptide-2 and the regulation of intestinal growth and function. Compr Physiol 8:1185-1210.

Brubaker PL, Izzo A, Hill M, and Drucker DJ (1997) Intestinal function in mice with small bowel growth induced by glucagon-like peptide-2. Am J Physiol 272: E1050-E1058. 
Bruens L, Ellenbroek SIJ, van Rheenen J, and Snippert HJ (2017) In vivo imaging reveals existence of crypt fission and fusion in adult mouse intestine. Gastroenterology 153:674-677.e3.

Buchman AL, Katz S, Fang JC, Bernstein CN, and Abou-Assi SG; Teduglutide Study Group (2010) Teduglutide, a novel mucosally active analog of glucagon-like peptide-2 (GLP-2) for the treatment of moderate to severe Crohn's disease. Inflamm Bowel Dis 16:962-973.

Compher C, Gilroy R, Pertkiewicz M, Ziegler TR, Ratcliffe SJ, Joly F, Rochling F, and Messing B (2011) Maintenance of parenteral nutrition volume reduction, without weight loss, after stopping teduglutide in a subset of patients with short bowel syndrome. JPEN J Parenter Enteral Nutr 35:603-609.

Crenn P, Coudray-Lucas C, Thuillier F, Cynober L, and Messing B (2000) Postabsorptive plasma citrulline concentration is a marker of absorptive enterocyte mass and intestinal failure in humans. Gastroenterology 119:1496-1505.

Dong CX, Zhao W, Solomon C, Rowland KJ, Ackerley C, Robine S, Holzenberger M, Gonska T, and Brubaker PL (2014) The intestinal epithelial insulin-like growth factor-1 receptor links glucagon-like peptide-2 action to gut barrier function. Endocrinology 155:370-379.

Drucker DJ, DeForest L, and Brubaker PL (1997) Intestinal response to growth factors administered alone or in combination with human $\left[\mathrm{Gly}^{2}\right]$ glucagon-like peptide 2. Am J Physiol 273:G1252-G1262.

Drucker DJ, Erlich P, Asa SL, and Brubaker PL (1996) Induction of intestinal epithelial proliferation by glucagon-like peptide 2. Proc Natl Acad Sci USA 93 7911-7916.

Dubé PE, Forse CL, Bahrami J, and Brubaker PL (2006) The essential role of insulinlike growth factor- 1 in the intestinal tropic effects of glucagon-like peptide- 2 in mice. Gastroenterology 131:589-605.

Galipeau HJ, Rulli NE, Jury J, Huang X, Araya R, Murray JA, David CS, Chirdo FG, McCoy KD, and Verdu EF (2011) Sensitization to gliadin induces moderate enteropathy and insulitis in nonobese diabetic-DQ8 mice. J Immunol 187:4338-4346.

Hadjiyanni I, Li KK, and Drucker DJ (2009) Glucagon-like peptide-2 reduces intestinal permeability but does not modify the onset of type 1 diabetes in the nonobese diabetic mouse. Endocrinology 150:592-599.

Hargrove DM, Alagarsamy S, Croston G, Laporte R, Qi S, Srinivasan K, Sueiras-Diaz J, Wisniewski K, Hartwig J, Lu M, et al. (2020) Pharmacological characterization of apraglutide a novel long-acting peptidic glucagon-like peptide-2 agonist for the treatment of short bowel syndrome. J Pharmacol Exp Ther DOI: 10.1124/jpet.119. 262238 [published ahead of print].

Iakoubov R, Lauffer LM, Trivedi S, Kim Y-I, and Brubaker PL (2009) Carcinogenic effects of exogenous and endogenous glucagon-like peptide-2 in azoxymethanetreated mice. Endocrinology 150:4033-4043.

Jeppesen PB (2013) Modern treatment of short bowel syndrome. Curr Opin Clin Nutr Metab Care 16:582-587.

Jeppesen PB, Gilroy R, Pertkiewicz M, Allard JP, Messing B, and O'Keefe SJ (2011) Randomised placebo-controlled trial of teduglutide in reducing parenteral nutrition and/or intravenous fluid requirements in patients with short bowel syndrome. Gut 60:902-914.

Jeppesen PB, Hartmann B, Thulesen J, Graff J, Lohmann J, Hansen BS, Tofteng F Poulsen SS, Madsen JL, Holst JJ, et al. (2001) Glucagon-like peptide 2 improves nutrient absorption and nutritional status in short-bowel patients with no colon Gastroenterology 120:806-815.

Jeppesen PB, Pertkiewicz M, Messing B, Iyer K, Seidner DL, O'keefe SJ, Forbes A Heinze H, and Joelsson B (2012) Teduglutide reduces need for parenteral support among patients with short bowel syndrome with intestinal failure. Gastroenterology 143:1473-1481.e3.

Kissow H, Hartmann B, Holst JJ, Viby NE, Hansen LS, Rosenkilde MM, Hare KJ, and Poulsen SS (2012) Glucagon-like peptide-1 (GLP-1) receptor agonism or DPP-4 inhibition does not accelerate neoplasia in carcinogen treated mice. Regul Pept 179: 91-100.

Koehler JA, Baggio LL, Yusta B, Longuet C, Rowland KJ, Cao X, Holland D, Brubaker PL, and Drucker DJ (2015) GLP-1R agonists promote normal and neoplastic intestinal growth through mechanisms requiring Fgf7. Cell Metab 21:379-391.

Lansing M, Turner JM, Wizzard P, Lavallee CM, Lim DW, Muto M, Nation PN, Pencharz PB, Ball RO, and Wales PW (2019) Plasma citrulline is not a biomarker for intestinal adaptation in short bowel syndrome, studied in piglets: a model for human neonates. Pediatr Surg Int 35:657-663.
Lee SJ, Lee J, Li KK, Holland D, Maughan H, Guttman DS, Yusta B, and Drucker DJ (2012) Disruption of the murine Glp2r impairs Paneth cell function and increases susceptibility to small bowel enteritis. Endocrinology 153:1141-1151.

Litvak DA, Hellmich MR, Evers BM, Banker NA, and Townsend CM Jr. (1998) Glucagon-like peptide 2 is a potent growth factor for small intestine and colon. $J$ Gastrointest Surg 2:146-150.

Marier JF, Mouksassi MS, Gosselin NH, Beliveau M, Cyran J, and Wallens J (2010) Population pharmacokinetics of teduglutide following repeated subcutaneous administrations in healthy participants and in patients with short bowel syndrome and Crohn's disease. J Clin Pharmacol 50:36-49.

Marietta E, Black K, Camilleri M, Krause P, Rogers RS III, David C, Pittelkow MR, and Murray JA (2004) A new model for dermatitis herpetiformis that uses HLADQ8 transgenic NOD mice. J Clin Invest 114:1090-1097.

Munroe DG, Gupta AK, Kooshesh F, Vyas TB, Rizkalla G, Wang H, Demchyshyn L, Yang Z-J, Kamboj RK, Chen H, et al. (1999) Prototypic G protein-coupled receptor for the intestinotrophic factor glucagon-like peptide 2 Proc Natl Acad Sci USA 96:1569-1573.

Ohneda K, Ulshen MH, Fuller CR, D'Ercole AJ, and Lund PK (1997) Enhanced growth of small bowel in transgenic mice expressing human insulin-like growth factor I. Gastroenterology 112:444-454.

Orhan A, Gögenur I, and Kissow H (2018) The intestinotrophic effects of glucagon-like peptide-2 in relation to intestinal neoplasia. J Clin Endocrinol Metab 103:2827-2837.

Ring LL, Nerup N, Jeppesen PB, Svendsen LB, and Achiam MP (2018) Glucagon like peptide-2 and neoplasia; a systematic review. Expert Rev Gastroenterol Hepatol 12 : $257-264$.

Rowland KJ, Trivedi S, Lee D, Wan K, Kulkarni RN, Holzenberger M, and Brubaker PL (2011) Loss of glucagon-like peptide-2-induced proliferation following intestinal epithelial insulin-like growth factor-1-receptor deletion. Gastroenterology 141 2166-2175.e7.

Seidner DL, Joly F, and Youssef NN (2015) Effect of teduglutide, a glucagon-like peptide 2 analog, on citrulline levels in patients with short bowel syndrome in two phase III randomized trials. Clin Transl Gastroenterol 6:e93.

Slim GM, Lansing M, Wizzard P, Nation PN, Wheeler SE, Brubaker PL, Jeppesen PB, Wales PW, and Turner JM (2019) Novel long-acting GLP-2 analogue, FE 203799 (apraglutide), enhances adaptation and linear intestinal growth in a neonatal piglet model of short bowel syndrome with total resection of the ileum. JPEN $J$ Parenter Enteral Nutr 43:891-898.

Tavares W, Drucker DJ, and Brubaker PL (2000) Enzymatic- and renal-dependent catabolism of the intestinotropic hormone glucagon-like peptide-2 in rats. Am J Physiol Endocrinol Metab 278:E134-E139.

Trivedi S, Wiber SC, El-Zimaity HM, and Brubaker PL (2012) Glucagon-like peptide2 increases dysplasia in rodent models of colon cancer. Am J Physiol Gastrointest Liver Physiol 302:G840-G849.

Tsai CH, Hill M, Asa SL, Brubaker PL, and Drucker DJ (1997) Intestinal growthpromoting properties of glucagon-like peptide-2 in mice. Am $J$ Physiol 273: E77-E84.

Ulshen MH, Dowling RH, Fuller CR, Zimmermann EM, and Lund PK (1993) Enhanced growth of small bowel in transgenic mice overexpressing bovine growth hormone. Gastroenterology 104:973-980.

Wiśniewski K, Sueiras-Diaz J, Jiang G, Galyean R, Lu M, Thompson D, Wang YC, Croston G, Posch A, Hargrove DM, et al. (2016) Synthesis and pharmacological characterization of novel glucagon-like peptide-2 (GLP-2) analogues with low systemic clearance. J Med Chem 59:3129-3139.

Yusta B, Holland D, Waschek JA, and Drucker DJ (2012) Intestinotrophic glucagonlike peptide-2 (GLP-2) activates intestinal gene expression and growth factordependent pathways independent of the vasoactive intestinal peptide gene in mice. Endocrinology 153:2623-2632.

Yusta B, Huang L, Munroe D, Wolff G, Fantaske R, Sharma S, Demchyshyn L, Asa SL, and Drucker DJ (2000) Enteroendocrine localization of GLP-2 receptor expression in humans and rodents. Gastroenterology 119:744-755.

Address correspondence to: Dr. P.L. Brubaker, Departments of Physiology and Medicine, University of Toronto, Rm. 3366, Medical Sciences Bldg., 1 King's College Circle, Toronto, ON M5S 1A8, Canada. E-mail: p.brubaker@ utoronto.ca 